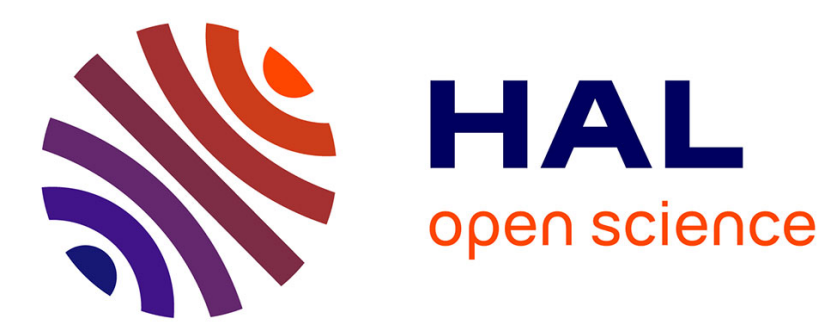

\title{
Dette, stratégies matrimoniales et institution d'héritier : sur l'élite paysanne lombarde au IXe siècle
}

\author{
Laurent Feller
}

\section{To cite this version:}

Laurent Feller. Dette, stratégies matrimoniales et institution d'héritier : sur l'élite paysanne lombarde au IXe siècle. Revue historique, 2008, 645 (2), p. 339-368. halshs-00355859

\section{HAL Id: halshs-00355859 \\ https://shs.hal.science/halshs-00355859}

Submitted on 25 Jan 2009

HAL is a multi-disciplinary open access archive for the deposit and dissemination of scientific research documents, whether they are published or not. The documents may come from teaching and research institutions in France or abroad, or from public or private research centers.
L'archive ouverte pluridisciplinaire HAL, est destinée au dépôt et à la diffusion de documents scientifiques de niveau recherche, publiés ou non, émanant des établissements d'enseignement et de recherche français ou étrangers, des laboratoires publics ou privés. 


\section{DETTE, STRATEGIES MATRIMONIALES ET INSTITUTION D'HERITIER : SUR L'ELITE PAYSANNE LOMBARDE AU IX ${ }^{\mathrm{e}}$ SIECLE.}

Laurent FELLER

Université Paris I Panthéon-Sorbonne/LAMOP

Notre connaissance des élites paysannes du haut Moyen Âge s'est considérablement renforcée depuis la décennie 1990. Les travaux de Chris Wickham et ceux de Jean-Pierre Devroey ont ouvert la voie à de nouvelles approches d'histoire sociale qui ont permis d'abord d'identifier, à la tête des collectivités rurales, des individus ou des groupes se distinguant par leur statut juridique, leur fonction ou leur richesse. Placée au point de jonction entre l'aristocratie, définie par son leadership politique et par sa richesse foncière, et la paysannerie entendue de façon très extensive comme le groupe des producteurs ou des cultivateurs, cette élite joue un rôle déterminant dans l'organisation sociale et politique de l'époque carolingienne $^{1}$. Elle exerce une fonction essentielle de médiation entre les sociétés locales et la société englobante et, sans son existence et sa relative efficacité, l'organisation même de l'État carolingien aurait été encore plus problématique qu'elle ne le fut. Jusqu'à un certain point, il existait en effet des moyens de transmettre ordres et informations du souverain vers le bas de la société ou, à une autre échelle, des seigneurs vers la paysannerie. La qualification juridique du groupe est certes importante : la participation aux institutions publiques, l'armée et le plaid, joue un rôle dans la distinction. Elle n'est cependant pas l'unique critère à retenir : durant le IX ${ }^{\mathrm{e}}$ siècle, il est évident que la participation aux opérations militaires était devenue le fait de l'aristocratie, de plus en plus spécialisée dans les tâches guerrières. L'élite paysanne n'en a pas moins continué à jouer un rôle et, surtout, des dynamiques permettant son renouvellement ont perduré durant toute la fin de l'époque carolingienne, malgré les difficultés de tous ordres que le groupe paysan rencontrait alors à cause de la rapidité et, parfois, de la brutalité du processus de transformation sociale et économique en cours ${ }^{2}$. Ces

\footnotetext{
${ }^{1}$ Jean-Pierre Devroey, L'espace des échanges économiques. Commerce, marché, communications et logistique dans le monde franc au $\mathrm{IX}^{\mathrm{e}}$ siècle, dans Uomo e spazio nell'alto medioevo, Spolète $\left(50^{\mathrm{a}}\right.$ settimana di studio del CISAM), 2003, p. 347-395

${ }^{2}$ Jean-Pierre Devroey, Puissants et misérables. Système social et monde paysan dans l'Europe des Francs $\left(V T^{e}\right.$ $I X^{e}$ siècles), Bruxelles, 2006 Académie royale de Belgique, p. 331 sv. Chris Wickham, Framing the Early Middle Ages. Europe and the Mediterranean (400-800), Oxford, Oxford University Press, 2005, p. 535-541, p. $570-578$
} 
difficultés aboutissent à restreindre l'autonomie de la paysannerie, voire, dans certains cas, à la faire disparaître totalement, par son insertion plus ou moins forcée dans des réseaux de clientèle ou de dépendance aristocratique dont le développement entraîne le transfert du contrôle des procédures économiques aux grands détenteurs de terre. Cela n'avait pas été le cas jusqu'alors, sauf dans les régions d'implantation massive du grand domaine, c'est-à-dire essentiellement en Gaule du Nord.

Pour illustrer ces propos, je me propose, après avoir rappelé quelques-unes des règles régissant la circulation des richesses au sein de la paysannerie, de présenter le dossier d'un groupe familial paysan italien, celui des Leopegisi. Je présenterai leur fortune en la comparant aux autres fortunes paysannes de même niveau connues, puis étudierai leur activité économique telle qu'elle résulte des documents donnant des informations sur les emprunts et les échanges, pour terminer par l'analyse un processus de dépossession qui n'allait pas nécessairement de soi.

\section{Les échanges fonciers au IX siècle : le comportement des élites paysannes.}

En Italie, qu'il s'agisse de l'Italie centrale ou de l'Italie septentrionale, le groupe paysan est bien documenté durant l'ensemble du IX siècle. Les chartes nous montrent le fonctionnement d'une société dont le fondement économique est la petite exploitation et nous dévoilent les stratégies mises en œuvre par les acteurs pour assurer la reproduction de leur groupe $^{3}$. Les membres de l'élite sont d'abord des propriétaires fonciers plus importants que les autres, même lorsque leurs possessions sont limitées à un et un seul terroir, ce qui n'est au demeurant pas toujours le $\mathrm{cas}^{4}$. Ils se distinguent de bien d'autres manières, par leur comportement économique comme par leur rapport à l'écrit. Mais les élites paysannes se définissent aussi, en Italie du moins, par la facilité avec laquelle elles accèdent à l'écrit pour valider les transactions foncières qu'elles effectuent, sans préjudice des autres rites qui viennent donner une forme à l'échange et le parachever. Tout transfert de quelque importance passe par l'établissement d'une charte, même aux niveaux sociaux les plus bas. Or, les actes qui nous sont parvenus ne disent pas toute la réalité des opérations réalisées : c'est ce point que nous nous proposons d'étudier ici, à travers l'analyse d'une brève série de documents

\footnotetext{
${ }^{3}$ Laurent Feller, Agnès Gramain et Florence Weber, La fortune de Karol. Marché de la terre et liens personnels dans les Abruzzes au haut Moyen Âge, Rome (Collection de l'EFR, n³47), 2005.

${ }^{4}$ Sur le caractère polynucléaire des possessions de certains membres de l'élite paysanne, voir Wendy Davies, Small Worlds. The Village Community in Early Medieval Brittany, Los Angeles, University of California Press, 1988 , p. 86. Ce point fait problème : ce caractère est en effet commun avec l'aristocratie, dont l'un des critères de définition est bien, à cette époque mais aussi encore au $\mathrm{XI}^{\mathrm{e}}$ siècle, la multiplicité des possessions sur une aire plus ou moins vaste.
} 
faisant principalement alterner des donations et des ventes portant parfois sur un même bien, suscitant de ce fait par moment une impression d'incohérence formelle. L'hypothèse à examiner et à prendre en compte est cependant celle d'une mobilisation d'une forme (la vente ou la donation) en fonction d'intentions précises qu'il faut essayer d'éclaircir. Parmi elles, celle de l'emprunt et du gage occupe une place importante.

Trois champs d'investigation et de réflexion sont apparus presque immédiatement : l'investissement, les politiques matrimoniales, l'organisation des successions. C'est sur ces trois facteurs, rarement présents tous ensemble dans un même dossier, que l'attention doit se porter afin d'éclairer la dynamique sociale à l'œuvre. Il existe toutefois un certain nombre de précautions. La bibliographie de la question montre, par exemple, la complexité de la notion d'investissement. Celui-ci en effet se réalise essentiellement à travers le marché de la terre, par l'achat et la vente de parcelles ou d'exploitations, dans un contexte juridique formel qui reconnait la plénitude de la propriété privée. Il n'en demeure pas moins que l'achat comme la vente sont des opérations susceptibles de recevoir des interprétations divergentes voire contradictoires : des achats qui, à première vue semblent relever de la pure concentration foncière apparaissent ainsi, après analyse, être liés à la politique matrimoniale des agents qui, en procédant à des échanges de terre, même contre de l'argent, préparent en fait des alliances de mariage, parfois des années avant que celles-ci ne deviennent effectives ${ }^{5}$. La nature même de 1'objet échangé, de la terre, contraint à quelque circonspection. Il est rare, en effet, mais il n'est pas tout à fait impossible, que, dans la société italienne du haut Moyen Âge, on soit en présence d'échanges marchands, dans lesquels les choses échangées sont totalement distinctes des gens qui les échangent: la terre continue, normalement, même après la transaction, et quelle que soit la forme de celle-ci, don ou vente, de conserver quelque chose de son ancien propriétaire. Cette considération, qui relève désormais du truisme, complique beaucoup les choses lorsque, par derrière ces échanges, se profile la question du crédit.

La question des liquidités est l'une des questions fondamentales que pose le problème de l'investissement, qu'il soit effectué dans des buts économiques, constituer ou accroître son revenu, ou dans des buts sociaux, accroître l'importance et la puissance de son réseau d'amitié en multipliant les alliances de mariage. L'emprunt peut être contracté autant pour acheter un bien de production - comme une terre - ou d'infrastructure - comme un moulin - que pour

\footnotetext{
${ }^{5}$ Laurent Feller, Introduction. Enrichissement, accumulation et circulation des biens. Quelques problèmes liés au marché de la terre dans Le marché de la terre au Moyen Âge, Laurent Feller et Chris Wickham éd., Rome, (Collection de 1'Ecole française de Rome, $n^{\circ} 350$ ), 2005 p. 3-28.
} 
préparer les mariages des enfants et, en milieu lombard, plus spécifiquement, des garçons puisque ceux-ci doivent détenir une exploitation viable au moment de leur mariage ${ }^{6}$.

La solution passe par l'emprunt sur gage foncier, selon des modalités jadis analysées par Cinzio Violante, dans des pages qui n'ont pas été contestées ni reprises ${ }^{7}$. Les prêts à intérêt, encore ouvertement avoués à la fin du VIII ${ }^{\mathrm{e}}$ siècle, ne le sont plus après 806 et le capitulaire de Nimègue ${ }^{8}$. Celui-ci, définissant le femus, le gain légitime et l'opposant au turpe lucrum, assimilé à l'avaritia et à la cupiditas, permettait la restriction du champ de légitimité du prêt à intérêt ${ }^{9}$. Les agents ont donc mis au point des techniques assez sophistiquées de prêts dissimulés derrière des ventes fictives ou, plus simplement, derrière des transferts fictifs de propriété, quelle qu'ait pu en être la forme. Lorsque ces opérations tournent mal, c'est-à-dire lorsque le débiteur ne peut pas rembourser, ses terres passent à son créancier, parfois un monastère et, avec elles, les titres de propriété. Pour cette raison, nous ne connaissons en général que des opérations qui se sont terminées par la faillite économique et sociale du débiteur ${ }^{10}$.

La mise en circulation des terres peut donc répondre à plusieurs objectifs différents : accroître son patrimoine pour accroitre la taille de son exploitation, construire pour ses fils des exploitations d'attente, s'endetter ou, au contraire, rembourser sa dette. Ces objectifs ne sont pas contradictoires : une logique de recherche d'alliance contraint à faire les mêmes choses qu'une simple logique d'accumulation. Suivant le moment de la vie, et les acteurs mis en cause par les échanges, l'action revêt plusieurs significations, parfois imbriquées les unes dans les autres, ce qui ne facilite évidemment pas leur interprétation: s'endetter pour se procurer une terre, ce peut-être à la fois pour investir, pour préparer l'établissement d'un fils, mais aussi pour resserrer des liens d'amitié avec le vendeur, lesquels peuvent très bien déboucher, au demeurant, sur une alliance de mariage...

\footnotetext{
${ }^{6}$ Régine Le Jan, Aux origines du douaire médiéval $\left(\mathrm{VI}^{\mathrm{e}}-\mathrm{X}^{\mathrm{e}}\right.$ siècle), dans Veuves et le veuvage dans le haut Moyen Age, M. Parisse éd., Paris, Picard, 1993, p.107-122 [repris dans Ead., Femmes, pouvoir et société dans le haut Moyen Âge, Paris, Picard, 2001, p. 53-67] ; Laurent Feller, Morgengabe, dot, tertia : rapport Introductif, dans Dots et douaires dans le haut Moyen Âge, François Bougard, Laurent Feller et Régine Le Jan éd., Rome (Collection de l'Ecole française de Rome, 295), 2002, p. 1-25.

Cinzio Violante, Les prêts sur gage foncier dans la vie économique et sociale de Milan au $\mathrm{XI}^{\mathrm{e}}$ siècle, Cahiers de Civilisation Médiévale, 5, 1962, p. 147-168 et 437-459. Id, Per lo studio dei prestiti dissimulati in territorio milanese ( secoli X-XI) , dans Studi in onore di Amintore Fanfani, I, éd., Milano, 1962, p. 641-735.

${ }^{8}$ Voir par exemple Codice Diplomatico Longobardo, n' ${ }^{\circ}$ XIX, col. 128-129. Erminald prête à Jean, fils d'Aretheo prête 900 deniers d'argent (75 sous), une somme évidemment importante. Un intérêt, labor, est prévu, stipulé en nature.

${ }^{9}$ MGH, Karoli Magni Capitularia, I, p. 182.

${ }^{10}$ Une exception remarquable, cependant, dans le dossier de Pierre de Niviano, un notable qui ne dédaigne pas, dans les années 870 , de pratiquer le prêt. C'est l'un des rares cas où 1'on ait le dossier complet d'un acteur gagnant. François Bougard, Pierre de Niviano, dit le Spolétin, sculdassius, et le gouvernement du comté de Plaisance à l'époque carolingienne, Journal des Savants, juillet-décembre1996 , p. 291-337.
} 
Dans la plupart des cas, les opérations qui viennent d'être décrites passent par l'achat et la vente, qui peuvent être soldées en numéraire ou en nature, donner lieu ou non à une estimation de la valeur, passer par une mesure de la surface ou non. Là encore, selon le but poursuivi, et selon la scène sociale à l'intérieur de laquelle l'action se déroule, la vente prend des formes elles-mêmes différentes ${ }^{11}$. Une même forme, un acte de vente, peut ainsi documenter une transaction purement marchande, passant par une mesure de la superficie et une estimation de la valeur, ou décrire une transaction visant à ouvrir une relation en instituant une dette, par exemple en sous-évaluant volontairement le bien vendu ou en l'échangeant contre un bien dont la valeur ne correspond manifestement pas à celle de la terre cédée $^{12}$.

Il n'est absolument pas nécessaire que les acteurs passent par la vente. La donation est également utilisée, entre égaux statutaires, pour organiser la circulation des terres et pour établir des relations de dépendance entre les parties. Elle est particulièrement pratique pour masquer les relations de crédit. Elle est en tout cas, dans ces circonstances, à l'opposé du don maussien, puisque c'est celui qui reçoit un bien de valeur qui détient alors le prestige et, vraisemblablement, la richesse ${ }^{13}$. Mais naturellement, la forme de la donation n'est pas univoque et les circonstances de son utilisation sont nombreuses: une grammaire systématique de son emploi est encore à écrire ${ }^{14}$.

\section{Les Leopegisi de Cologno Monzese.}

Le dossier de textes dont il va être question concerne une famille d'alleutiers lombards, celle des Leopegisi, vivant à Cologno Monzese, entre Monza et Milan au IX siècle. Elle est documentée sur 40 années, de 842 à 882 par une dizaine de textes conservés dans le fonds documentaire de Saint-Ambroise de Milan, riche pour le IX ${ }^{\mathrm{e}}$ siècle $^{15}$. Nous la

\footnotetext{
${ }^{11}$ Laurent Feller, Agnès Gramain, Florence Weber, La fortune de Karol, op. cit. (n. 3), p. 131-141.

${ }^{12}$ Sur ces variations, mise au point pratique dans Caroline Dufy et Florence Weber, L'ethnographie économique, Paris, 2007, p. 46-54.

${ }^{13}$ Voir Florence Weber, L'Essai sur le Don : vers une ethnographie des prestations sans marché, introduction à M. Mauss, Essai sur le Don, Paris, 1924 [éd. 2007, Presses Universitaires de France].

${ }^{14}$ Dans les Abruzzes, au IX ${ }^{\mathrm{e}}$ siècle, la donation, que ce soit à une institution ou à un saint ou à un égal statutaire, n'est jamais utilisée, seule la vente 1'étant. Laurent Feller, Autour de la fondation de San Clemente a Casauria: la constitution d'un patrimoine foncier à la fin du $\mathrm{IX}^{\mathrm{e}}$ siècle, dans Montecassino. Dalla prima alla seconda distruzione, Momenti e aspetti di storia cassinese, (secc.VI-IX). Atti del II Convegno di studi sull'alto Medioevo meridionale (Cassino-Montecassino, 27-31 maggio 1984), F. Avagliano éd., Abbazia di Montecassino, 1986, p. 513-526. Id., Les Abruzzes médiévales. Territoire, économie et société en Italie centrale du IX au XII siècle, Rome (Bibliothèque des Écoles françaises d'Athènes et de Rome, $\mathrm{n}^{\circ} 300$ ) 1998, p. 167-180.

15 Tous les actes en sont édités dans Porro-Lambertenghi, Historiae Patriae Momimenta, t. XIII, Codex Diplomaticus Langobardiae, Turin, 1873. Le plaid de 865 est donné par Cesare Manaresi, I placiti del Regnum Italiae, t. I, p. 242-246, $\mathrm{n}^{\circ}$ 67. Sur Saint-Ambroise, voir: Ross Balzaretti, The politics of property in ninth-
} 
suivons sur quatre générations et disposons d'informations assez précises sur son patrimoine et sur la façon dont celui-ci a été transmis, ainsi que sur son évolution à la fin de la période.

Cet ensemble de textes a déjà fait l'objet d'un commentaire approfondi de la part de Gabriella Rossetti en 1968, dans son étude classique sur la localité de Cologno Monzese ${ }^{16}$. Le cas des Leopegisi ne constitue qu'une partie minime de l'analyse mais apparaît exemplaire à bien des égards. G. Rossetti s'intéresse dans son travail à un phénomène de substitution d'élites articulée sur un bouleversement des structures foncières et agraires. La domination franque, en imposant le grand domaine, principalement dans les zones les plus riches de 1'Italie septentrionale, détruit littéralement l'élite des possessores lombards. C'est dire que, dans le courant du $\mathrm{IX}^{\mathrm{e}}$ siècle, le grand domaine remplace une structure foncière dominée par la petite et la moyenne propriété. Ici, l'acteur central et le principal bénéficiaire de la concentration foncière est le monastère de Saint-Ambroise dont la seigneurie tend à devenir hégémonique sur ce territoire, les moines devenant les seuls propriétaires fonciers d'importance à l'intérieur de la localité.

Si l'on suit la démonstration de Gabriella Rossetti, les Leopegisi se sont peu à peu appauvris. Ils ont dû emprunter de l'argent en se servant de leurs terres comme de gages. Leur créancier, Saint-Ambroise a fini par rafler la mise et s'approprier l'ensemble de leurs terres. G. Rossetti a placé au cœur de son étude la question du déclin d'une famille tout entière, victime de la politique d'expansion de la seigneurie ambrosienne, cas de figure du renforcement général de la puissance de l'aristocratie italienne au IX ${ }^{\mathfrak{e}}$ siècle. Il est en effet indéniable que les Leopegisi s'affaiblissent et s'appauvrissent. Mais cette analyse peut être dépassée sur un point qui me semble essentiel: la famille et ses membres ne sont pas demeurés sans réagir et ne se sont pas laissé absorber sans résister. Des politiques ont été élaborées, qui avaient pour but de préserver la richesse et le statut du groupe. Celles-ci sont allées très loin, puisqu'elles sont passées par la modification des règles de l'héritage et par l'institution d'un héritier préférentiel, chargé de sauvegarder les intérêts du groupe.

Saint-Ambroise de Milan est un monastère de fondation relativement récente, dont 1'histoire est liée à celle de la domination carolingienne sur l'Italie. C'est en 789 que

century Milan. Familial motives and monastic strategies in the village of Inzago, Mélanges de l'Ecole Française de Rome, Moyen Âge, 111/2, 1999 [= Les transferts patrimoniaux en Europe occidentale, VIII ${ }^{e}-X^{e}$ siècle], p. 747770 ; Gabriella Rossetti, Il monastero di S. Ambrogio nei primi due secoli di vita ; i fondamenti patrimoniali e politici della sua fortuna, dans Il monastero di S. Ambrogio nel Medioevo, Giorgio Picasso éd., Milan, 1988, p. 20-34 .

${ }^{16}$ Gabriella Rossetti, Società e istituzioni nel contado lombardo durante il medioevo, Cologno Monzese : $i$ secoli VIII-X, Milano, 1968, p. 101-122. 
Charlemagne, quinze années après la conquête de 1'Italie, l'a institué et doté ${ }^{17}$. Le monastère prend la succession d'une basilique qui abritait les reliques du saint. Cet acte, par lequel le souverain franc établit un lien entre l'ancien pouvoir romain et lui-même, lui donne aussi un levier lui permettant d'agir sur un territoire et sa société ${ }^{18}$. En réalité, Saint-Ambroise végète. La ressource idéologique qu'il pourrait représenter n'est pas mobilisée sur le champ et l'établissement met un certain temps à s'enrichir et à devenir un acteur de premier plan dans la vie économique locale. Son rôle ne devient effectivement majeur que vers 850 , sous le règne de Louis II, qui y est enterré ${ }^{19}$.

À partir de 850, le monastère intervient fréquemment sur le marché de la terre en achetant des biens. Il est présent aussi dans les circuits du crédit et prête de l'argent, soit directement, soit par l'intermédiaire d'hommes de paille. C'est là un point tout à fait essentiel dans la démonstration de $\mathrm{G}$. Rossetti qui soutient la thèse que le monastère, acteur obstiné et ne reculant devant aucun obstacle pour constituer sa seigneurie, agit sur tous les fronts de la vie économique locale. Elle pense, en particulier, que derrière toutes les opérations foncières de Leopegisi se cachent des prêts sur gage foncier consentis par le monastère à travers un truchement, le clerc Pierre. Elle étaie solidement sa démonstration mais, surtout, a recours à l'argument d'autorité massif que propose la bibliographie de Cinzio Violante. Il nous faut, avant tout, reparcourir le dossier et suivre, après G. Rossetti, les étapes des acquisitions et des ventes avant de juger la nature de la politique suivie par les Leopegisi.

\footnotetext{
17 Sur Saint-Ambroise, outré 1'article de R. Balzaretti cité à la note 14 voir, de prochaine parution, Ross Balzaretti, The Lands of Saint Ambrose. Monks and Society in Early Medieval Milan, Turnhout, Brepols, 2008.

${ }^{18}$ Sur des fondations de cette nature dans le monde franc : Mathw Innes, Kings, Monks and Patrons: Political Identities and the Abbey of Lorsch, dans La royauté et les élites dans l'Europe carolingienne, Régine Le Jan éd., Villeneuve d'Ascq, Centre d'Études d'Histoire de 1'Europe du Nord-Ouest, 1998, p. 301-324 ; Matthew Innes, State and Society in the Early Middle Ages. The Middle Rhein Valley, 400-1000, Cambridge, Cambridge University press (Cambridge studies in Medieval life and thought. 4th series). Pour le contexte italien et la signification des fondations royales ou princières : Barbara Rosenwein, The Family Politics of Berengar I, King of Italy (888-924), Speculum, 71, 1996, p. 247-289 ; Suzanne Wemple, S. Salvatore/S. Giulia : A Case Study in the Endowment and Patronage of a Major Female Monastery in Northern Italy, dans Women of the Medieval World: Essays in Honor of John H. Mundy, Julius Kirshner et Suzanne Wemple éd., Oxford et New York, Blackwell, 1985, p. 85-102 ; Laurent Feller, Les politiques des familles aristocratiques à l'égard des églises en Italie centrale ( $\mathrm{IX}^{\mathrm{e}}-\mathrm{XI}^{\mathrm{e}}$ siècles), dans Sawver son âme et se perpétuer. Transmission du patrimoine et mémoire du haut Moyen Âge, François Bougard, Cristina La Rocca et Régine Le Jan éd., Rome (Collection de 1'École française de Rome $\left.n^{\circ} 351\right)$ 2002, p. 265-292.

${ }^{19}$ Ross Balzaretti, The politics of property in Milan, cité à la note 14 .
} 


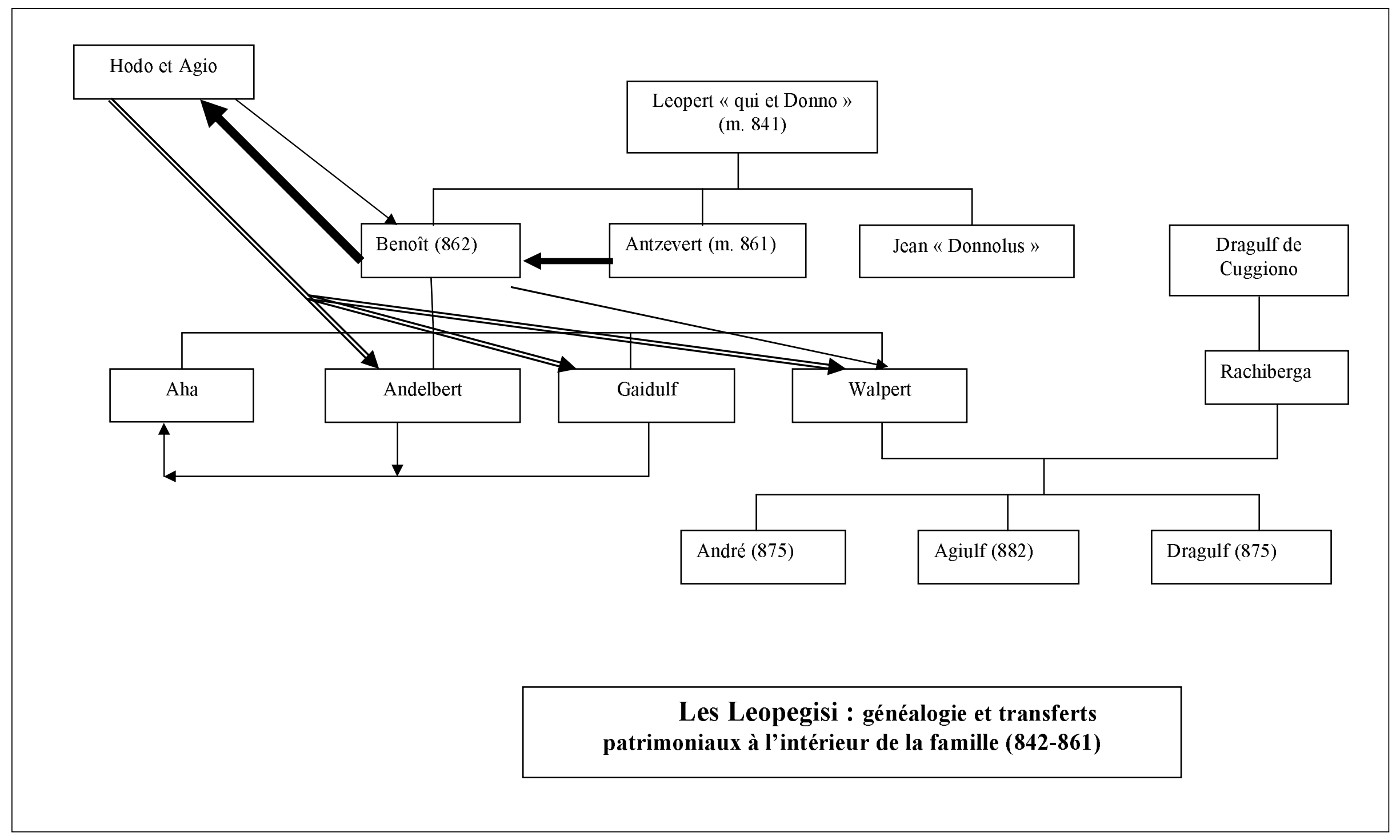


La chronologie des mouvements fonciers et la typologie des actes employés doit avant toute autre chose être décrite. Plusieurs actions se déroulent en même temps et s'entrecroisent entre 842 et 882 , les années 861-865 étant manifestement cruciales dans l'histoire du patrimoine et de la famille.

- Le premier document qui nous soit parvenu date de mai 842. C'est une donation effectuée par Benoit fils de Leopert au profit du clerc Pierre, originaire du bourg d'Albariate. Il lui cède une terre ainsi qu'un moulin tenu en livello par le prêtre. Benoît reçoit un launegild, un contre-don, consistant en une paire de gants (launechild manicias par uno).

- Vingt ans se passent, puis en mars 861, une nouvelle donation est effectuée, dont les motivations sont problématiques. Deux personnages, par ailleurs totalement inconnus, Hodon et Agion, donnent à Benoît et à ses fils Andelbert, Gaidulf et Walpert, l'ensemble des biens qu'ils avaient achetés à Benoît. Benoît reçoit l'usufruit du tiers de ses biens. Andelbert et Gaidulf se voient attribuer la propriété du tiers des biens de leur père. Quant à Walpert, il reçoit les biens de son oncle Antzevert, précédemment acquis par Benoît. Le sort patrimonial de la fille de celui-ci, Aha, est alors réglé par l'octroi d'une rente et la donation de quelques biens fonciers.

- Deux mois plus tard, en mai 861, Benoît, Walpert et Andelbert, le père et deux de ses fils, agissant conjointement, procèdent à une permutation de biens avec l'abbé de Saint-Ambroise. L'affaire porte sur un demi-hectare, environ, de terres et de prés.

- En décembre de la même année 861, Walpert et Gaidulf vendent pour 40 sous un moulin au clerc Pierre.

- Au début de 862, l'un des frères, Andelbert, ayant donné l'ensemble de ses biens au monastère de Saint-Ambroise, son prévôt à Cologno et Gaidulf procèdent à une division de cette propriété.

- En juillet 863, le clerc Pierre, acquéreur en 861 du moulin appartenant à Walpert et Gaidulf, le cède à Saint-Ambroise, comme gage dans le règlement d'un litige foncier portant sur des terres sises à Milan.

- Conséquence de cette cession, en janvier 865, un procès oppose Walpert à l'abbé de SaintAmbroise. Le conflit porte sur des terres occupées et mises en valeur par le premier, alors que l'abbaye en est propriétaire, au titre de la donation de juillet 863 . Walpert perd son procès et sort de scène.

- En février 875, André fils de Walpert vend à l'abbé, au prix de 5 sous, un droit de préemption sur l'ensemble de ses biens.

- Le même mois, sa mère, Rachiberga, veuve de Walpert, vend la moitié de sa Morgengabe au même abbé, au prix de 60 sous.

- L'année suivante, en mai 876, deux autres fils de Walpert et Rachiberge, mineurs, vendent, pour cause de famine, deux petites pièces de terre au prix de 8 sous.

- Enfin, en 882, Gaidulf, fils de Benoît, vend au monastère deux pièces de terre au prix de 7 sous.

Chronologie des affaires des Leopegisi entre 842 et 882

\begin{tabular}{|l|l|l|}
\hline Réf $^{20}$. & Date & Action \\
\hline Man. 67 & Mai 842 & $\begin{array}{l}\text { Donation de terres et d'un moulin au clerc Pierre par } \\
\text { Benoît }\end{array}$ \\
\hline CDL, CCXIV & Mars 861 & $\begin{array}{l}\text { Donation-partage des biens de Benoît par Hodon et } \\
\text { Agion }\end{array}$ \\
\hline
\end{tabular}
${ }^{20}$ Références : Man.= C. Manaresi, I Placiti del Regnum Italiae, I, Rome, 1955. CDL $=$ Codice Diplomatico
Longobardo. 


\begin{tabular}{|c|c|c|}
\hline$C D L$, CCXVI & Mai 861 & $\begin{array}{l}\text { Echange entre Benoît, Walpert et Andelbert et l'abbé } \\
\text { de Saint-Ambroise }\end{array}$ \\
\hline Man. 67 & Décembre 861 & $\begin{array}{l}\text { Vente d'un moulin au clerc Pierre par Walpert et } \\
\text { Gaidulf }\end{array}$ \\
\hline CDL, CCXXII & Mai 862 & Division des biens d'Andelbert et de Gaidulf \\
\hline CDL CCXXVI & Juillet 863 & $\begin{array}{l}\text { Donation du moulin à Saint-Ambroise par le clerc } \\
\text { Pierre }\end{array}$ \\
\hline Man 67 & Janvier 865 & Procès entre Walpert et Saint-Ambroise \\
\hline $\begin{array}{l}\text { CDL, CCLX et } \\
\text { Man. } 67\end{array}$ & Février 875 & $\begin{array}{l}\text { Vente à Saint-Ambroise par André d'un droit de } \\
\text { préemption sur ses biens }\end{array}$ \\
\hline$C D L$, CCLXI & Février 875 & $\begin{array}{l}\text { Vente à Saint-Ambroise par Rachiberge de la moitié } \\
\text { de sa Morgengabe }\end{array}$ \\
\hline$C D L$, CCLXVII & Mai 876 & Vente de biens par Agiulf et Dragulf \\
\hline$C D L, \mathrm{CCCXV}$ & 882 & Vente de biens à Saint-Ambroise par Gaidulf \\
\hline
\end{tabular}

Tableau récapitulatif

De cette séquence, le patrimoine des Leopegisi sort considérablement transformé et affaibli, et la position sociale des membres de la famille s'en trouve affectée. La thèse que nous allons soutenir est que les Leopegisi ne sont pas restés inertes face au monastère et à sa politique foncière agressive, mais que, au contraire, ils ont développé une politique financière et foncière destinée à lui faire pièce.

Etre riche en milieu rural au IX siècle : consistance et évaluation d'un patrimoine.

\section{Eléments matériels}

Les Leopegisi sont, entre 840 et 860, engagés dans des affaires nombreuses. Possédant au moins un moulin, on peut les classer sans l'ombre d'un doute parmi l'élite villageoise définie par sa seule richesse. Ils ne détiennent aucun pouvoir et rien ne dit qu'ils aient cherché à en obtenir. Bref, si ce sont des notables, leur pouvoir est limité au seul vicus de Cologno. Ils entretiennent cependant des rapports à l'extérieur de leur village, le clerc Pierre étant originaire d'une autre localité et étant également un acteur sur le marché foncier à l'intérieur de Milan. Tardivement, et certainement pas pour leur profit, ils entrent également en relation avec Saint-Ambroise. On a là des personnages qui dominent peut-être économiquement leur communauté, mais qui sont en situation d'infériorité dès lors qu'ils traitent en dehors de celleci. Par ailleurs, leur participation réelle aux institutions publiques n'est pas établie : il est impossible de savoir s'ils sont encore convoqués au plaid ou s'ils se rendent encore à l'armée. Cela semble peu probable: ils se maintiennent en permanence en-dessous de ce seuil de notabilité. 
La documentation conservée donne une idée de la fortune de cette famille parce que des éléments de celle-ci sont alors minutieusement décrits.

En mars 862, un partage a lieu entre Gaidulf, fils de Benoît, et le prévôt de SaintAmbroise. Il fait suite à un acte qui n'a pas laissé de trace, la donation de tous ses biens au monastère par Andelbert, fils de Benoit ${ }^{21}$. Or, Andelbert est dans 1'indivis avec son frère Gaidulf. Il est alors nécessaire de procéder à une répartition des terres appartenant à chacun des deux frères.

La donation faite à Saint-Ambroise apparaît comme l'occasion de séparer matériellement les parts afin que le monastère puisse entrer en jouissance de la sienne. On sait que chacun des deux hommes possède à ce moment le tiers des biens fonciers de leur père. Ce document offre ainsi l'opportunité d'examiner la totalité du patrimoine de l'un des acteurs, Andelbert, puisqu'il a donné tout son avoir, et de se rendre compte de ce que représente chacune des parts d'héritage. Comme il s'agit de partages effectués par parts précisément définies, cette division reflète avec exactitude l'intégralité des biens dont il est question ${ }^{22}$. Comment cela se présente-t-il ?

Concrètement, la division se fait pièce par pièce, selon les repères que donnent les points cardinaux : les limites sont fixées en fonction du nord (de monte), du sud (meridione), de l'est (de mane) et de l'ouest (de sera). Chacune des deux parties emporte l'un des deux côtés de la division principale : si le monastère prend la partie occidentale de la pièce, Gaidulf conserve la partie orientale. Il n'y a pas d'orientation privilégiée. Ce mode opératoire implique une certaine imprécision, à moins d'admettre que les champs sont bornés, ce qui est peu probable : les confronts sont destinés à demeurer flous, ce qui pose problème. Comment imaginer en effet que le monastère puisse installer un tenancier qui ne soit pas l'ancien propriétaire dans une propriété dont les limites ne seraient pas définies avec exactitude ? Dans la mesure où les autres ayants-droit, c'est-à-dire les héritiers potentiels frustrés par la donation faite au monastère, peuvent, de surcroît éprouver quelque ressentiment de la nouvelle situation, la conclusion logique est que, malgré la donation, Andelbert est demeuré sur place avec son frère. Toute autre combinaison serait difficilement viable.

Les biens concernés sont constitués de 34 items divisés entre deux maisons, deux vignes, 17 champs, trois prés, une châtaigneraie, deux rouvraies, trois fragments d'insulae, c'est-à-dire vraisemblablement des lieux aptes à recevoir des moulins, cinq pièces de taillis

\footnotetext{
${ }^{21} \mathrm{CDL}, \mathrm{n}^{\circ} \mathrm{CCXXII}$, col. 370-373.

${ }^{22}$ Les droits de la sœur de Gaidulf et d'Andelbert, appelée Aha, sur les biens de ses frères sont réservés avec toute la précision nécessaire. Nous verrons plus loin par quels moyens.
} 
(stallaria). La superficie totale n'en est pas connue, seules quelques rares pièces de terre étant mesurées.

La maison (casa sala) doit être d'une certaine importance. Les termes utilisés invitent à penser qu'il s'agit d'un manoir auquel sont attachés des éléments de prestige et de pouvoir social : ce pourrait être une sorte de «hall», une maison dotée d'un lieu de réception rapprochant la résidence d'une demeure seigneuriale. Elle est divisée en deux parts égales, aucune compensation n'étant organisée en ce qui la concerne. Il y a sans doute là une indication sur la valeur accordée par chacune des parties à la possession de la maison. Indirectement, cela pourrait également signifier que les Leopegisi ont un statut au moins de coq de village, comme l'indique le surnom donné au père de Benoît, Donno, et le diminutif du frère de celui-ci, Donnolus, commentés par G. Rossetti qui y voit le signe de 1'aisance de la famille. Ces gens ont en tout cas un certain prestige social et leur fortune les place au-dessus des autres habitants.

La division énumère ensuite des terrains spécialisés, des cours, des prés, des vergers puis des terres arables. Les parcelles arables dominent l'ensemble, par le nombre (17 parcelles). La seule mention de production céréalière dont nous disposions dans ce dossier se trouve dans une rente versée en seigle et en panic ${ }^{23}$.

La description permet de restituer la structure du bien. Il se présente comme une exploitation dont les différentes composantes sont complémentaires, et qui permet de pratiquer une polyculture vivrière assez diversifiée. On note en particulier une forte présence de l'inculte et des surfaces boisées. Une châtaigneraie, pour la farine et le bois d'œuvre, une chênaie (roboretum), pour l'élevage porcin, le bois d'œuvre et le bois de chauffe, des taillis, pour le petit bois et la dépaissance des bestiaux. Des prés, enfin, qui doivent être des prés de fauche et dont la présence est liée à l'abondance de l'eau dans cette région. Le dernier élément à commenter est constitué par la présence d'insulae. Ce ne sont pas nécessairement des îles, mais des pièces de terre proches de l'eau et partiellement au moins entourées par une rivière : elles sont donc propices, soit à l'installation de pêcheries, soit à la construction de moulins.

La division respecte la structure de l'exploitation, chaque élément, sauf une vigne échangée contre un pré, étant divisé en deux parties égales. Les indications de superficie sont, quant à elles, très peu nombreuses. Elles concernent quelques prés (l'un mesurant 30 tables, l'autre 100 tables $)$, la rouvraie $(24 \text { tables })^{24}$ et une parcelle de vigne. Celle-ci est très petite

\footnotetext{
${ }^{23}$ CDL XIII, $n^{\circ}$ CCXIV, col. 352a.

${ }^{24}$ On retient les équivalences proposées par François Menant dans sa thèse : François Menant, Campagnes lombardes du Moyen Age. L'économie et la société murales dans la région de Bergame, de Crémone et de
} 
puisqu'elle est estimée à 12 tables, soit $336 \mathrm{~m}^{2}$. Elle est échangée contre un pré de 31 tables, soit $868 \mathrm{~m}^{2}$. On pourrait avoir là une sorte d'équivalence entre deux éléments patrimoniaux, la vigne ayant une valeur nettement plus grande que les prés. Il est difficile d'exploiter systématiquement ces données. L'impression qui ressort, toutefois, est celle d'un parcellaire éclaté et d'une exploitation qui, très rationnellement, se déploie sur plusieurs terroirs.

Nous disposons, toujours concernant cette famille, de quelques autres documents permettant de nous représenter la disposition du parcellaire. En 861, Benoît et deux de ses fils ont procédé à une permutation de biens avec l'abbé de Saint-Ambroise. Celui-ci cède un champ enclos d'une superficie de deux perches, soit $1340 \mathrm{~m}^{2}$ et un pré d'environ 150 tables, soit $4260 \mathrm{~m}^{2}$, ce qui fait au total $5600 \mathrm{~m}^{2}$, mais obtient en contrepartie un pré d'un jugère, soit $8000 \mathrm{~m}^{2}$. Toutes les pièces obtenues par les laïcs jouxtent des communaux, ce qui est sans doute la raison de l'échange. Il ne s'agit pas là de superficies considérables : l'impression donnée par la lecture de la division est sans doute la bonne. Le paysage est constitué de micro-parcelles de terres arables et de parcelles plus conséquentes de prés, et l'exploitation s'appuie sur des biens communaux. Il est possible enfin que, à la génération de Benoît, la famille soit sur une sorte de frontière, celle séparant l'exploitant direct, le cultivateur, de l'exploitant indirect, le rentier du sol. L'hypothèse selon laquelle, à la génération de Benoît, la famille ne travaille pas elle-même, mais dirige 1'exploitation est, au vu de ce patrimoine, acceptable. La position de la famille est cependant fragile si aucune acquisition n'est faite et si les partages successoraux sont effectués selon la loi.

L'élément le plus important de cette fortune est le moulin, possédé par la famille de 842 à décembre 861. Le partage de 861 en a attribué la moitié à Walpert, Gaidulf et Andelbert se partageant l'autre moitié. Au moment de la division entre l'abbé et Gaidulf, il est déjà sorti des biens familiaux. La possession du moulin a assuré pendant un temps une rente à la famille. Sa cession, en décembre 861, apparaît comme une forme de liquidation de la fortune familiale, dont les difficultés éventuelles sont accrues par la donation d'Andelbert au monastère.

\section{Eléments immatériels}

Brescia du Xe au XIII siècle, Rome (Bibliothèque des Écoles françaises d'Athènes et de Rome, n²81), 1993, p. $797-802$.

\begin{tabular}{|l|l|}
\hline 1 table & $28 \mathrm{~m}^{2}$ \\
\hline 1 perche & $670 \mathrm{~m}^{2}$ \\
\hline 1 jugère & $8000 \mathrm{~m}^{2}$ \\
\hline
\end{tabular}


Il est possible d'estimer non pas la valeur des terres mais celle de la fortune de cette famille, ce qui inclut presque nécessairement aussi des éléments immatériels. Il faut pour cela examiner une autre transaction, celle par laquelle Rachiberga, épouse de Walpert, cède sa Morgengabe à l'abbé de Saint-Ambroise ${ }^{25}$.

On sait que, au moment du mariage, les femmes lombardes reçoivent une série de biens $^{26}$. Le père fournit une dot (faderfio), normalement en liquide ou en tout cas en biens mobiliers. La fille n'est pas pour autant exclue de l'héritage sur lequel elle a des droits, certes moins importants que ceux de ses frères, mais réels. Son mari, lui, doit deux prestations à son épouse. La première, la meta, est, depuis le VIII ${ }^{\mathrm{e}}$ siècle, versée directement à la femme. Par ce transfert, le mari acquiert le mund sur son épouse, c'est-à-dire le droit de la commander et le devoir de la protéger, ainsi que les droits sur sa capacité à hériter. La seconde est le don du matin, la Morgengabe, qui vient récompenser la virginité de la fille et rémunérer l'ensemble des gratifications sexuelles à venir ${ }^{27}$. Il s'agit du quart de ses biens, offert en toute propriété. À la mort du mari, la femme a le droit d'en disposer comme elle l'entend, sous couvert de ses fils si elle en a. Normalement, toutefois, c'est sur cette fraction des biens de son mari que sont assis les biens de la veuve : elle n'a donc pas intérêt à s'en dessaisir, sauf cas de nécessité absolue ou si elle doit changer de statut. Un remariage est en effet souvent accompagné de la cession la Morgengabe, afin de marquer la fin de toute relation entre la famille du premier mari et la femme ${ }^{28}$. La vente de la Morgengabe est un acte grave, parce qu'elle prive la femme de ce qui doit normalement lui permettre d'entretenir son statut. Cet acte a nécessairement des conséquences autres que la simple acquisition d'argent: il est très vraisemblable que l'acheteur ne soit pas quitte pour le prix, mais qu'il soit moralement tenu de veiller désormais sur la veuve, comme si en acquérant la terre ou une part de celle-ci, il devait aussi prendre en charge une partie du mund. Les héritiers naturels, qui sont privés de 1'espérance de récupérer ce bien à la disparition de leur mère, se trouvent également dans une position particulière à l'égard du monastère.

Ici, Rachiberga vend la moitié de sa Morgengabe au prix de 60 sous, versés en deniers, bonos et novos, chaque sou contenant 12 deniers précise le texte : il s'agit bel et bien d'une

\footnotetext{
${ }^{25}$ CDL XIII, $n^{\circ}$ CCLXI, col. 439-440.

${ }^{26}$ Jack Goody, L'évolution de la famille et du mariage en Europe, Paris, 1986, p. 251; Laurent Feller, Morgengabe, dot, tertia ... dans Dots et douaires..., op.cit. (note 6).

${ }^{27}$ Diana Owen Hugues, From Brideprice to Dowry in Mediterranean Europe, Journal of Family History, 1978, p. $262-296$.

${ }^{28}$ Laurent Feller, Autour des archives du Mont-Cassin et du cartulaire de Pierre Diacre : la Morgengabe de Iesulfa, comtesse de Teano, dans Retour aux sources. Textes, études et documents d'histoire médiévale offerts à Michel Parisse, Sylvain Gouguenheim, Monique Goullet et Laurent Morelle éd., Paris, Picard, 2003, p. 473484.
} 
transaction monétarisée. Cela permet une esquisse de calcul et d'avoir au moins un ordre de grandeur. À partir de cette donnée chiffrée, on infère que la totalité du patrimoine de Walpert au moment de son mariage peut avoir été estimé à 480 sous. C'est une très grosse somme qui n'est cependant pas improbable à ce niveau social. La composition maximale, le hériban, est, pour sa part de 600 sous tandis que 500 sous constituent l'ordre de grandeur des investissements réalisés au $\mathrm{IX}^{\mathrm{e}}$ siècle par les plus gros opérateurs repérables ${ }^{29}$. On ne s'attend pas à ce qu'une telle somme soit réalisable, la liquidité des fortunes étant très limitée. On rapprochera ces montants (les 60 sous de la Morgengabe et les 480 d'évaluation de la totalité du patrimoine) des 40 obtenus pour le moulin en 862 . On a là l'image d'une fortune moyenne dans l'Italie lombarde de la seconde moitié du IX ${ }^{\mathrm{e}}$ siècle.

Si ces approximations contiennent une part de vérité, on doit considérer que la famille, peut-être en cours d'appauvrissement, a encore du bien. Elle n'est pas alors, dans les années 870, en voie d'être prolétarisée, même si elle connaît des problèmes du fait de l'insertion du monastère dans ses affaires et du fait de l'érosion violente qui s'est produite en 861 et 862 avec la vente du moulin d'une part et, de l'autre, la donation de tout son bien au monastère par Andelbert, ce qui, au bout du compte, représente le 1/3 du patrimoine de Benoît. La famille a cependant mobilisé tous les moyens à sa disposition pour éviter un fractionnement rapide de son patrimoine. En revanche, la conservation de son statut à la tête de la communauté villageoise, qui ne va pas de soi, passe très certainement par l'insertion dans la clientèle de l'abbaye : dans la deuxième moitié du VIII ${ }^{\mathrm{e}}$ et au début du $\mathrm{IX}^{\mathrm{e}}$ siècle, en Toscane, à Campori dans la Garfagnana, les Gundualdi n'ont pas agi différemment ${ }^{30}$, et, pour consolider leur position, les membres de ce groupe familial ont délibérément choisi de s’insérer dans le réseau des évêques de Lucques.

\section{Recherche de comparaisons : situations analogues au}

IX siècle

Grâce aux dossiers étudiés ou réétudiés depuis une dizaine d'années, nous disposons de plusieurs points de comparaisons. Nous évoquerons trois cas, à titre de comparaison : la famille des Totoni, Pierre de Niviano et Karol fils de Liutprand ${ }^{31}$.

\footnotetext{
${ }^{29}$ Laurent Feller, Les Abruzzes médiévales..., op. cit. (note 14), p. 187-190.

${ }^{30}$ Chris Wickham, The Mountains and the City. The Tuscan Appennines in the Early Middle Ages, Oxford, Clarendon Press, 1988, p. 40-51.

${ }^{31}$ Les monographies sur des individus ou des familles sont de plus en plus nombreuses. Outre les exemples développés par Chris Wickham, que ce soit dans The Mountains and the City cité à la note précédente ou dans d'autres de ses ouvrages comme Comunità et clientele (Chris Wickham, Comunità e clientele nella Toscana del XII secolo. Le origini del comune rurale nella Piana di Lucca, Roma, Viella, 1995 [trad. française, Communautés et clientèles en Toscane au XIT siècle. Les origines de la commune rurale dans la région de
} 
Le groupe familial des Totoni, installé au VIII siècle sur les bords du lac de Lugano semble avoir été sensiblement plus riche que les Leopegisi ${ }^{32}$. Les Totoni possèdent davantage de terres, détiennent des esclaves et, surtout, ne sont à aucun moment des cultivateurs directs. Leurs revenus semblent construits en partie autour du commerce de l'huile dont ils approvisionnent Milan. S'ils ne peuvent être classés dans un groupe aristocratique, c'est parce qu'ils n'ont aucune participation au pouvoir, à quelque niveau que ce soit. De plus, la concentration en un seul lieu de leur fortune foncière les prive de l'un des éléments caractéristiques des fortunes aristocratiques, la multipolarité des possessions et leur dispersion sur une aire régionale assez vaste. Les Leopegisi appartiennent manifestement à un degré inférieur de la société lombarde, du fait de la moindre importance de leurs possessions et de l'absence d'orientation vers le marché de leur production.

Pierre de Niviano, dit le Spolétin, présente pour sa part une fortune d'un niveau apparemment semblable aux Leopegisi. Les renseignements sur celles-ci, et sur sa gestion, sont plus nombreux, le dossier comportant une gamme beaucoup plus vaste de documents : alors que les Leopegisi n'ont laissé que des titres de propriété, Pierre de Niviano a ainsi transmis des actes de gestion, livelli et constitutions de prêts. Il semble que sa fortune soit liée à sa fonction de sculdassius ${ }^{33}$, c'est-à-dire à sa position d'agent public de niveau inférieur. Pierre est d'implantation récente et il construit son patrimoine au fur et à mesure de son long séjour comme officier dans la localité de Niviano. C'est un autre cas de figure, celui d'une notabilité liée autant à la fonction politique qu'à la fortune héritée ou construite : il est, pour reprendre une terminologie de la sociologie rurale, hors-groupe. Les Leopegisi, pour leur part, sont en-groupe, c'est-à-dire qu'ils ne cessent pas d'appartenir au groupe paysan dans lequel ils peuvent à tout moment retourner si leurs opérations ne sont pas couronnées de succès. Là encore, les Leopegisi sont un cran en-dessous de Pierre. Ils n'ont pas de participation au pouvoir; ils ne sont pas susceptibles de prendre autant d'initiatives économiques que ce dernier qui, de surcroît, est 1'un des rares cas documentés de réussite à ce niveau social au IX siècle. Pierre de Niviano est en effet parvenu à construire, gérer et stabiliser son patrimoine au moment où il s'est retiré des affaires. C'est l'absence d'héritier qui l'a amené à céder

Lucques, 2001, s. 1. (Bibliothèque d'Histoire rurale, 2)], il faut également citer 1'étude de K. Bullimore: Katherine Bullimore, Folcwin of Rankweil : the world of a Carolingian local officer, Early Medieval Europe, 13, 1, 2005, p. 43-77 ; La position de Folcwin présente des caractères analogues à celle de Pierre de Niviano.

${ }^{32}$ Carte di famiglia. Strategie, rappresentazione e memoria del gruppo familiare di Totone di Campione (721877), Stefano Gasparri et Cristina La Rocca éd., Rome, Viella, 2005.

${ }^{33}$ François Bougard, Pierre de Niviano..., op. cit. (note 10). 
l'ensemble de ses biens à la cathédrale de Plaisance, après une vie fructueuse et, du point de vue patrimonial, bien menée.

De ce point de vue, les Leopegisi sont semblables à la famille de Karol fils de Liutprand. Les sommes d'argent que les Leopegisi manipulent, les placent cependant très audessus du niveau économique et social de 1'Abruzzais, bien que les procédures mises en œuvre et les problèmes rencontrés soient sensiblement les mêmes ${ }^{34}$. Karol procède en effet durant sa vie active, entre 840 et 870 environ, à une vingtaine d'achats mais ne dépense qu'une quarantaine de sous. Après sa mort, ses héritiers sont amenés, dans des circonstances que l'on ne peut présenter ici, à céder leurs terres au monastère impérial nouvellement fondé de S. Clemente a Casauria et à entrer dans sa clientèle en reprenant ces mêmes terres en précaire. Le fait économique qui a précipité le déclin de la famille de Karol est un emprunt que ses héritiers n'ont pas pu rembourser. La raison pour laquelle cet emprunt avait été contracté est la constitution d'exploitations d'attente pour les couples formés par chacun de ses trois fils. C'est là encore l'ampleur des sommes en jeu qui différencie les deux dossiers. Mais il existe entre eux des points de convergence, puisque, dans l'un et l'autre cas, des familles d'alleutiers rencontrant des difficultés financières sont contraintes de traiter avec un monastère de fondation récente dont le patrimoine est encore en cours de constitution. Les terres familiales sont absorbées par l'établissement religieux et les membres de la famille agrégés à son réseau de dépendants. Cependant, alors que la crise de la famille de Karol est liée à des calculs partiellement erronés et à des erreurs d'appréciation dans le choix des alliances matrimoniales, dans le cas des Leopegisi, le facteur premier des difficultés semble bien être l'accroissement de la pression foncière exercée par Saint-Ambroise.

Au cœur des deux dossiers, cependant, se trouvent deux questions, celle de l'emprunt sur gage foncier, d'une part, et celle du mariage des fils, d'autre part. Sous-tendant les comportements des deux groupes familiaux, le désir d'une ascension sociale est manifeste. Karol a orienté les mariages de ses fils pour la rendre possible à la génération suivante. Il n'y a que partiellement réussi, mais les raisons mêmes qui entraînent son échec relatif se retrouvent dans le dossier des Leopegisi. On ne peut manquer d'avancer l'hypothèse selon laquelle Leopert aurait réussi à consolider sa position économique. À la différence de Pierre de Niviano, il ne s'est pas appuyé sur une position politique. En revanche, il a sans doute bénéficié de la faiblesse de la concurrence et des pressions de gros propriétaires fonciers. Ses

\footnotetext{
${ }^{34}$ Laurent Feller, Agnès Gramain, Florence Weber, La fortune de Karol, op. cit. (note 3), p. 55-58.
} 
fils, et plus encore ses petits-fils ne parviennent pas, pour leur part, à se maintenir au même niveau social et économique. Le trajet, ascendant jusqu'au début des années 840 , cesse de l'être dès la seconde génération. Il en va sans doute de même pour Karol fils de Liutprand, dont les héritiers sont incapables de regagner les positions atteintes par leur père et qui sombrent rapidement.

\section{Dons et gages, emprunts et échanges dans le processus d'appauvrissement}

Il est certain que le groupe familial connaît des difficultés. Celles-ci semblent liées aux affaires construites autour du moulin et à la relation établie entre le clerc Pierre et les Leopegisi $^{35}$. En 842, Pierre reçoit un don de la part de Benoît, qui agit alors conjointement avec son frère Jean. Le transfert de propriété concerne des terres situées le long de la rivière Lambro: ce sont des terres de rives susceptibles de recevoir un moulin. De fait, Pierre annonce son intention de construire là des écluses, c'est-à-dire les dérivations nécessaires au fonctionnement de moulins. Benoît et Jean cèdent aussi, par le même acte, la propriété d'un moulin sur le Lambro, tenu en livello par le prêtre Jean. En échange, ils ne reçoivent qu'une paire de gants, c'est-à-dire le launegild nécessaire à parfaire la transaction. Il s'agit d'une contrepartie qui n'a rien à voir avec une quelconque valeur.

L'acte est difficile à interpréter parce qu'il ne comporte aucun exposé des causes qui le provoquent: simple donation entre vifs, qui sont apparemment des égaux statutaires, elle n'est pas explicitée comme le serait une donation faite à un établissement pieux pro remedio anime. Ce pourrait être un simple gage d'amitié, une action de générosité destinée à renforcer la position morale de son auteur et donc son rang à l'intérieur de la communauté ${ }^{36}$. Le don d'une terre périphérique de l'exploitation et sur laquelle aucun investissement n'est prévisible dans l'immédiat de la part du propriétaire peut être un moyen de resserrer les liens d'amitié à l'intérieur de la collectivité et d'assurer au donateur, Benoît, une forme de supériorité sur le bénéficiaire, Pierre. La réciprocité doit jouer en effet : l'objet offert en contre don, une paire de gants, n'efface pas réellement l'obligation de rendre, mais est le témoignage d'un lien durable établi par la donation. Autrement dit Pierre, qui a donné des objets mobiliers de faible valeur économique contre des biens fonciers potentiellement de forte valeur et contre la propriété d'une rente, est en dette à l'égard de Benoît. Le launegild, alors, ne doit pas être considéré comme un contre don, mais comme un gage qui garantit la bonne exécution d'un

\footnotetext{
${ }^{35}$ G. Rossetti, Società..., op. cit. (note 16) p. 108-114.

${ }^{36}$ Pour une théorie des actes de cette nature, voir C. Wickham, Framing the Middle Ages, op. cit. (note 2) p. 535 Sv.
} 
contrat dont les termes ne sont pas explicites en liant les personnes et en matérialisant une obligation $^{37}$. La donation est donc, par la forme même qu'elle prend, un contrat qui exige une contrepartie: au reste la loi lombarde le précise bien, puisqu'elle prévoit que dans les donations faites aux établissements pieux, on ne doit ni attendre ni exiger de thinx ou de launegild, précisément parce qu'il s'agit de dons faits à Dieu ou à ses saints et qu'ils n'ont de valeur que s'ils sont gratuits ${ }^{38}$.

Ce n'est pas ce qui se produit. La nature de l'obligation du clerc Pierre à l'égard de Benoît n'est pas précisée. S'agissant de personnages qui sont, malgré la cléricature de Pierre, des égaux statutaires, mais ne sont ni parents ni alliés, elle ne peut-être que d'ordre économique et l'hypothèse d'un prêt octroyé par Pierre à Benoît est, dans ces conditions, la plus vraisemblable. Elle change la signification même de l'acte. Dans le cas d'un don, Benoît acquiert un pouvoir sur Pierre, parce que celui-ci est en dette à son égard tant qu'il n'a pas rendu le don. Si, au contraire, le texte ne décrit pas la totalité de l'échange et si les gants signifient l'obligation pour Benoît de rendre autre chose, en l'occurrence de 1'argent, la relation est inverse et c'est évidemment Pierre qui se trouve en position de supériorité, parce que créancier.

Le recours au don et la mobilisation du système rituel qui l'entoure, permettent d'éviter la vente. Les raisons de ce choix ne sont pas très claires. Toutefois, dans la société lombarde du IX ${ }^{\mathrm{e}}$ siècle, la vente confirmée par écrit garantit la stabilité de la propriété et le caractère définitif de l'échange. L'un des actes du dossier le dit au détour de son formulaire. Dans la permutation de possessions organisée en 861 entre Benoît, ses fils et l'abbé de SaintAmbroise, la notification de l'acte se présente ainsi: « qu'il soit connu qu'un contrat d'échange [a été dressé] de bonne foi, afin qu'il ait la solidité de 1'achat ${ }^{39}$ ». Il doit être plus difficile de revenir sur une vente que sur toute autre forme de mutation. Surtout, celle-ci limite les possibilités de constitution d'obligation une fois le prix versé. La situation est donc très différente de celle observée dans les Abruzzes où, au même moment, les ventes de biens fonciers servent à ouvrir des relations qui sont assimilables à des relations de crédit : le jeu sur le montant des prix ou sur les contreparties exigées dans l'échange autorise l'établissement ou la création d'obligations que seul un examen attentif du contexte de l'opération permet de

\footnotetext{
${ }^{37}$ M. Mauss, Essai sur le don ... op. cit. (note 13), p. 250-254.

${ }^{38}$ Sur le launegild, sa signification et son évolution, voir Chris Wickham, Compulsory gift-exchange in Lombard Italy (650-1150), à paraître dans Wendy Davies et Paul Fouracre éd., The language of gift. Je remercie vivement C. Wickham de m'avoir fait lire son texte et de m'avoir autorisé à le citer.

${ }^{39} \mathrm{CDL} \mathrm{n}^{\circ} \mathrm{CCXVI}$, col. $359 \mathrm{~d}$ : Commutatio bone fidei noscitur esse contractum, ut vicem emcionis optineat firmitatem.
} 
reconstituer. Le recours à la donation et à son rituel de remise d'un lannegild, en revanche, place immédiatement les acteurs dans le même registre, mais avec plus de facilité. Entre égaux statutaires, c'est sans doute un moyen plus souple et peut-être même plus efficace que le jeu des ventes successives, lequel présente une difficulté supplémentaire, celle de l'existence et du maintien de la confiance entre les parties, puisqu'il n'est pas possible de faire intervenir le juge en cas de conflit. Autrement dit encore, l'apparence de donation permet de masquer la réalité de l'échange marchand en cours de réalisation. L'emprunt se trouve alors encastré dans un processus d'échange non marchand et occulté par lui, parce que «la chose elle-même, donnée et engagée dans le gage, est, par sa vertu propre, un lien ${ }^{40} »$.

\section{La nouvelle répartition des biens à l'intérieur de la famille en 861}

Il reste alors à comprendre la suite de la séquence et, pour cela, suivre strictement la chronologie. Il nous faut remonter au début du dossier, au transfert opéré en mars 861 de Hodon et Agion vers Benoît et ses héritiers. Son effet, nous le verrons plus bas, est de répartir différemment le patrimoine familial ${ }^{41}$. Plusieurs actions se déroulent donc simultanément : il est nécessaire d'en examiner le détail. Hodon et Agion, qui ne semblent pas appartenir à la parenté des Leopegisi, ont acquis par achat, dit le texte, l'ensemble des biens de Benoît. Le prix n'est pas indiqué. De leur libre volonté, mais sans indiquer la raison, ils transfèrent tous ces biens, à titre gratuit, aux trois fils de celui-ci, selon des modalités que l'on a déjà évoquées mais qu'il faut maintenant préciser. Notons immédiatement que la donation est close par la transmission d'un launegild, encore une fois une paire de gants. I1 indique que la relation ouverte par l'achat fait à Benoît n'est pas close et que, si la fratrie reçoit des terres et se voit confirmée dans son héritage, elle a sans doute des devoirs à l'égard de Hodon et de Agion. L'hypothèse qui vient immédiatement à l'esprit est celle d'un transfert de la dette du père à ses fils, conjointement responsables du remboursement. Dès lors, la distribution du patrimoine a pour fonction d'assurer celui-ci, même si le résultat est la construction d'une nouvelle organisation familiale. Ici, la relation de crédit va extrêmement loin, puisqu'elle permet au créancier d'intervenir dans la vie même d'un groupe familial en son entier. Il faut cependant que le consentement des Leopegisi ait été acquis, c'est-à-dire que la pression du créancier ait rencontré la volonté de ses débiteurs.

Les biens se répartissent ainsi: Walpert reçoit l'intégralité des biens de son oncle Antzevert et se voit confirmer tout ce que son père a pu lui donner auparavant. Ses frères,

\footnotetext{
${ }^{40}$ Marcel Mauss, Essai sur le don, op. cit. (note 13), p. 253.

${ }^{41} \mathrm{CDL}, \mathrm{n}^{\circ} \mathrm{CCXIV}, \mathrm{col} .351-352$.
} 
Gaidulf et Andelbert, se voient attribuer chacun un tiers des biens de leur père, celui-ci gardant l'usufruit du tiers restant. Quant à Aha, la sœur, elle est simplement dédommagée, puisqu'elle reçoit une rente en nature, six muids annuels de panic et de seigle, assis sur les biens de ses frères, à condition toutefois qu'elle demeure célibataire. Andelbert remet le lannegild aux deux donateurs.

Benoît est peut-être le seul héritier de son père Leopertus. La part de son frère Antzevert lui est échue et il n'est pas impossible que celle du troisième frère, Jean dit «Donnolus », dont on ne sait rien, lui soit également parvenue. En tout cas, en 842, lors de la donation effectuée au profit de Pierre, Benoît a agi en son nom, un peu comme si celui-ci ne comptait pas. Nous ne connaissons de descendance ni à Antzevert ni à Jean. Il est assuré que le premier n'en a pas, puisque tous ses biens sont allés à Benoît. On ne peut exclure que Benoît ait reconstitué l'intégralité du patrimoine de son père Leopert, malgré la loi qui oblige à un partage égalitaire. À tout le moins, il a hérité de $2 / 3$ des biens de celui-ci.

L'un des points intéressants réside en ce qu Hodo et Agio ont commencé par régler la question de la succession d'Antzevert : ses biens sont attribués au seul Walpert. Benoît n'a donc pas constitué une masse unique, mais il a toujours distingué des parts individualisables par ses héritiers. Une ligne de succession d'oncle à neveu est alors établie. Elle est exclusive, puisque Walpert ne reçoit rien, à ce moment du moins, des biens de son père. Cependant, si Andelbert et Gaidulf venaient à disparaître sans héritiers, Walpert ou ses descendants parviendraient à reconstituer la totalité du patrimoine de Benoît, et peut-être de Leopert. Le partage privilégie évidemment Walpert qui se voit attribuer au moins le tiers des biens de son grand-père et peut-être la moitié de ceux-ci si la part de Jean «Donnolus » a été partagée entre Antzevert et Benoît, alors que ses frères n'en auront, à terme, qu'une fraction comprise entre le quart et le sixième. De plus, Hodo et Agio lui donnent la moitié du moulin familial. Celui-ci est divisé en deux parts égales dont l'une va à Walpert et la seconde à ses frères Andelbert et Gaidulf. Les biens cédés à Walpert sont donc manifestement plus importants que ceux obtenus par Andelbert et Gaidulf et, par conséquent, le revenu comme le prestige qu'il peut en retirer sont eux aussi supérieurs aux revenus et au prestige de ses frères.

Andelbert et Gaidulf reçoivent donc chacun un tiers des biens de leur père Benoît, le troisième étant laissé à celui-ci en usufruit et étant destiné à leur revenir à parts égales après son décès. Le destin de leur sœur, Aha, est également réglé : le document, comme on l'a déjà mentionné, organise son célibat. La fille n'est pas exclue de l'héritage, mais elle est ici maintenue dans une sujétion très étroite. D'une part, elle reçoit un logement (caminata cum vestiario suo), un jardin d'une superficie de huit tables, soit $225 \mathrm{~m}^{2}$, mais à titre d'usufruit 
seulement. Elle se voit attribuer, d'autre part, une rente viagère en grain. La jouissance de l'ensemble, la maison, la terre et le revenu, est subordonnée au maintien d'Aha dans le célibat. Si elle se marie, rien ne lui est dû au titre de la dot ou de l'héritage et elle perd son usufruit. Il est peu vraisemblable, dans ces conditions, que les prétendants se bousculent.

Une garantie existe toutefois: si ses frères ne paient pas la rente due, Aha aura la jouissance des biens dont son père a gardé l'usufruit, et ce jusqu'à sa mort. Ensuite, les terres reviendront à ses frères, equaliter abendum. La situation patrimoniale est donc absolument verrouillée : rien ne devrait pouvoir sortir du patrimoine des Leopegisi du fait de Aha. Enfin, on a le fort soupçon que Andelbert et Gaidulf ne sont pas non plus destinés à se marier et à procréer une descendance légitime. Il est possible, dans ces conditions, que des tensions soient nées au sein de la famille et la donation de l'intégralité de sa part à Saint-Ambroise par Andelbert en 862 pourrait en être le signe. Elle prive en tout cas Walpert de la possibilité de mettre la main sur les biens de celui-ci, tout en créant des difficultés pratiques pour Gaidulf qui doit partager une exploitation jusque là gérée dans l'indivision. Une autre hypothèse, d'ailleurs pas nécessairement incompatible, peut être avancée : Andelbert, incapable de faire face à ses engagements, aurait donné ses biens au monastère afin, peut-être, d'en conserver la jouissance. Dans les Abruzzes, les fils de Karol, affrontés à une situation similaire, ont transféré au monastère de Casauria les biens que leur créancier revendiquait, transformant ainsi l'établissement religieux en protecteur, au prix de leur statut : d'alleutiers ils devenaient alors tenanciers livellaires ${ }^{42}$. Les acteurs ne restent donc pas inertes ou passifs face à la pression d'un gros propriétaire foncier hégémonique. Ils réagissent et élaborent des stratégies qui leur permettent dans ce cas de retarder et dans d'autres sans doute d'éviter la perte de leur propriété et de leur indépendance. Le mécanisme à l'œuvre n'est pas sans parade: tout dépend de 1'habileté des acteurs à construire des stratégies et à les mettre en œuvre : cela nous emmène au-delà de 1'analyse de G. Rossetti qui, elle, pensait que ce déclin était inéluctable tant le rapport de forces était défavorable aux alleutiers.

Autant que l'hypothèse d'une dette dont le remboursement serait organisé dans la matérialité, il semble qu'il faille considérer celle d'une manœuvre destinée à freiner, voire à empêcher la fragmentation du patrimoine. Des parts inégales ont été définies et la plus importante a été attribuée à l'un des frères, que l'on a envie de qualifier d'aîné. L'organisation de ces transferts ne fait sens, cependant, que si les «cadets » acceptent d'être sacrifiés, ce qui en l'occurrence signifie ne pas se marier, afin que l'intégralité de la terre revienne à Walpert.

\footnotetext{
${ }^{42}$ Laurent Feller, Agnès Gramain, Florence Weber, La fortune de Karol, op. cit. (note 3), p. 106 sv.
} 
On a déjà trouvé trace de comportements de cette nature dans les Abruzzes au même moment $^{43}$. La constitution de maisons, c'est-à-dire d'entités dont la survie soit supérieure à celle des individus qui la composent, est en effet l'une des possibilités de défense que la paysannerie peut organiser dans une conjoncture délicate. Une telle organisation permet au moins de repousser les échéances et, peut-être, en assurant la viabilité de l'exploitation, de continuer à solder les dettes. Le résultat est d'avantager la maisonnée formée par Walpert et Rachiberga. Il n'est pas impensable que l'opération ait en fait été montée pour rendre possible ce mariage : ainsi, les frères de Walpert auraient été brimés pour constituer la Morgengabe de celle-ci, dont le poids en termes monétaires s'avère écrasant. Il est donc possible que la poursuite d'une stratégie d'ascension sociale ait inclus un mariage hypergamique et coûteux en termes monétaires comme en termes humains, et que la désignation d'un héritier préférentiel, d'un aîné ait été jugée nécessaire.

Un indice va dans le sens de la réalité de cette intention: en 882 , Gaidulf vend une pièce de terre à Saint-Ambroise. Il s'agit d'un sedimen d'une superficie de 12 tables $\left(336 \mathrm{~m}^{2}\right)$ et d'un pré de 60 tables $\left(1680 \mathrm{~m}^{2}\right)$, dont il obtient 7 sous, ce qui est beaucoup : il est vrai que la terre était enclavée dans celle du monastère ce qui a peut-être permis à Gaidulf de négocier son prix. Or, à la cession, signant juste après Gaidulf, se trouve mentionné un Agiulf, qui n'est autre que son neveu, le fils de Walpert. Agiulf a été consulté, même si son consentement n'est pas requis, parce que, fils de l'aîné, il avait des droits sur les biens de son oncle demeuré célibataire. En tout cas, à ce moment, en 882, Saint-Ambroise est devenu propriétaire hégémonique à Cologno et le destin de la famille des Leopegisi semble être effectivement de devoir décliner.

\section{Le processus d'aliénation des biens.}

Après le partage de mars 861 , les événements se succèdent à un rythme précipité durant quelques années. Le moulin de la famille est d'abord vendu, en décembre 861, au clerc Pierre au prix de 40 sous $^{44}$. S'agissant certainement du moulin en jeu dans l'acte de 842 , le but n'est pas de solder les comptes d'un emprunt, mais de se procurer des liquidités en un moment critique et, pour cela, de réaliser un bien d'importance pourtant cruciale pour la famille

\footnotetext{
${ }^{43}$ Ibid., p. $115 \mathrm{sv}$.

${ }^{44}$ Manaresi, I Placiti, no67, p. 244-245.
} 
Ce moulin, Pierre finit par le donner au monastère en $863^{45}$. G. Rossetti, à cause de cette dernière transaction voit en lui un homme d'affaires qui, dès le début, représente les intérêts du monastère et à travers lequel celui-ci a agi de façon continue depuis 842 . Cela n'est pas tout à fait exact: la donation du moulin par Pierre est effectuée dans le cadre d'un échange construit pour pacifier un violent conflit entre l'abbé de Saint-Ambroise et Pierre. L'acte rappelle au demeurant les origines de propriété : il y a eu effectivement deux cessions concernant le même bien, l'une en 842 , la donation, et l'autre en 862 , la vente. C'est cette dernière qui permet le transfert au monastère. La donation n'a donc pas eu d'effet réel durant tout ce temps là, signe que, s'il y a eu endettement, il y a eu aussi remboursement. Bien qu'ils n'en soient plus propriétaires, les deux frères Benoît et Jean ainsi que leurs descendants n'ont à aucun moment quitté physiquement la terre qu'ils mettaient en valeur et le moulin qu'ils exploitaient.

Le plaid de 865 est provoqué par le fait que Walpert, fils de Benoît est toujours sur place, malgré les transferts successifs de propriété. Il prétend être sur les terres de son oncle Antzevert, qui ne sont pas concernées, dit-il, par la vente de décembre 861. Les pièces apportées et lues au procès lui donnent tort : la vente du moulin, dans laquelle il était partie, portait effectivement aussi sur la part d'Antzevert. On entraperçoit alors l'une des raisons du partage de 861. Il s'agissait d'isoler la part de celui-ci afin de sauver des biens menacés en constituant un noyau dur de propriétés. La manœuvre échoue du fait de l'insertion de SaintAmbroise dans le processus. Si, avec Pierre, des négociations sont sans doute possibles, ce n'est pas le cas avec l'abbé qui, au moment où il cherche à consolider le patrimoine de son établissement a tout intérêt à se montrer intransigeant.

Le monastère se plaint de ce qu'il coupe des arbres, qu'il mette en culture des terres qui relèvent de la donation faite par le clerc Pierre et que, donc, il se soit lancé dans une opération de défrichement sur la terre sans l'accord du monastère qui crie alors à la spoliation et à l'usurpation. Walpert se comporte bien comme s'il continuait d'être effectivement propriétaire de la part de son oncle, ou comme détenait encore des droits d'exploitation, ce que le monastère conteste.

En 875, Rachiberga vend la moitié de sa Morgengabe à Saint-Ambroise et, le même mois, André, l'un des fils de Walpert, cède à Saint-Ambroise un droit de préemption : cela exclut désormais le recours à une aide extérieure, empêche tout nouvel emprunt sur gage

\footnotetext{
${ }^{45} \mathrm{CDL}$ XIII, $\mathrm{n}^{\circ} \mathrm{CCXVI}$, col. 377-380 (juillet 863).
} 
foncier et place de fait, sinon encore tout à fait de droit, cette branche de la famille dans la dépendance ou dans la clientèle de Saint-Ambroise.

\section{Conclusion}

Avec plus de détails, mais de nature différente, nous avons trouvé là un schéma déjà rencontré dans des dossiers concernant des alleutiers italiens et en particulier dans celui de Karol fils de Liutprand. Le premier point porte sur le caractère finalement secondaire du contexte général. Formellement, le système d'échanges de biens fonciers (achats, ventes, donations, permutation de biens) fonctionne. On ne voit pas d'évidentes spoliations. Toutefois, la présence d'un acteur surpuissant, en l'occurrence Saint-Ambroise, pèse sur l'ensemble, ne serait-ce, d'ailleurs, que parce que la sélection des documents s'est faite par son intermédiaire. Le fait que Saint-Ambroise ait mobilisé le plaid pour récupérer une terre aliénée au clerc Pierre montre qu'il est en mesure de casser tout système d'emprunts sur gages fonciers qui ne lui conviendrait pas : le dépouillement des Leopegisi de leur moulin est en réalité un effet induit, un dégât collatéral, dans un conflit opposant Pierre à Saint-Ambroise. Pour obtenir gain de cause, le premier a abandonné ses clients traditionnels.

Si nous admettons l'hypothèse de l'emprunt initial de Benoît auprès du clerc Pierre, la relation de crédit qui s'est alors établie entre les parties a couru sur le long terme : lorsque les Leopegisi ont eu besoin de liquidités de façon urgente, ils se sont retournés vers lui, seulement après, il est vrai, s'être mis dans une situation difficile et avoir accepté qu'un autre créancier exerce sur eux un pouvoir considérable et presque insupportable. Cette relation est établie non pas pour sauver provisoirement de la ruine une famille trop dépensière ou incompétente, mais pour rendre possible l'établissement des fils d'une part, et sans doute pour permettre la poursuite d'investissements productifs d'autre part. La constitution d'un patrimoine pour Walpert peut se ranger dans cette catégorie. Simplement, la capacité de financement de Benoît est excédée par sa deuxième opération. La vente du moulin en 861 est peut-être un appel lancé à un créancier habituel : le maintien de la présence de Walpert sur les lieux incite à penser que la vente a peut-être été, dans son intention, fictive mais que la cession du titre à l'abbé a interrompu un processus normal. Il n'en demeure pas moins que, malgré l'importance de leur patrimoine, les Leopegisi sont, structurellement, en position faible. Leurs stratégies semblent passer, au début des années 860, par des emprunts emboîtés - de la cavalerie - qu'ils rendent acceptables à leurs créanciers en modifiant les règles de dévolution patrimoniale au sein de leur famille. 
Les procédures utilisées sont tout à fait remarquables. Le recours à la donation scellée par le launegild nous place dans une sphère où échange marchand et échange non marchand se recouvrent et se recoupent. Les acteurs font plusieurs choses à la fois. Ils établissent des relations de crédit et des relations personnelles. Ils préparent des alliances de mariage et ils accroissent leur patrimoine. Les actes économiques analysés visaient donc à la fois à produire du rang, du prestige et de la richesse, c'est-à-dire du patrimoine et du revenu. Ils portent d'abord sur des moulins et sur des éléments de rente. Ils servent aussi à établir des alliances si les emprunts sont bien contractés pour rendre possibles des mariages. La recherche du gain, l'investissement, la construction des revenus sont présents: ils constituent des attitudes normales dans le groupe de l'élite paysanne, mais ils ne se dissocient pas de la construction des alliances de mariage et de la recherche d'une amélioration des statuts. On est là en présence de phénomènes ambivalents et inséparables, formellement, pour les acteurs comme pour l'enquêteur. Les investissements produisent aussi des effets sociaux, en fonction de leur réussite ou de leur échec, et les alliances de mariage sont autant des paris économiques autant que sociaux: la vente d'une partie de la Morgengabe de Rachiberga montre que l'incompétence en cette matière, ou tout simplement l'erreur d'appréciation, a un coût élevé.

Que nous apprend au bout du compte ce si riche dossier? Tout d'abord que les mécanismes sociaux à l'œuvre ne peuvent pas se réduire à un seul point, la ruine et la déchéance progressive des alleutiers. Certes, dans le cas analysé, il est bien probable que les choses aient fini par aller mal et que les Leopegisi, après avoir perdu la propriété de leur bien, aient aussi vu leur dignité atteinte après leur vraisemblable entrée dans la dépendance de Saint-Ambroise. Toutefois, on ne sait pas à quel niveau ils s'y sont agrégés : ils ont très bien pu continuer à jouir d'une forme de prestige social et à exercer une certaine domination sur leur entourage, tout en étant des clients de l'abbaye. S'ils ont conservé l'exploitation de leur terre, cela a certainement été le cas. D'autre part, la capacité de réaction qui est la leur, l'inventivité dont ils font preuve pour limiter les effets négatifs des problèmes qu'ils rencontrent, nous montrent un groupe qui est bien loin d'être anesthésié par les malheurs des temps et qui conserve encore quelques ressources morales et également sociales.

En d'autres termes, les difficultés du monde paysan, quoique réelles, ne sont pas si profondes qu'elles puissent détruire toutes les formes de sociabilité et tous les liens permettant l'organisation de la résistance à la déchéance. S'il y a bel et bien une crise du IX ${ }^{\mathrm{e}}$ siècle, elle ne se résout pas nécessairement par l'anéantissement de la paysannerie libre et par la dislocation de la société qu'elle forme jusqu'à la fin de l'époque carolingienne. 